\title{
Single-nucleotide resolution analysis of the transcriptome structure of Clostridium beijerinckii NCIMB 8052 using RNA-Seq
}

\author{
Yi Wang ${ }^{1,2}$, Xiangzhen $\mathrm{Li}^{3}$, Yuejian $\mathrm{MaO}^{3}$ and Hans P Blaschek ${ }^{2,45^{*}}$
}

\begin{abstract}
Background: Clostridium beijerinckii is an important solvent producing microorganism. The genome of C. beijerinckii NCIMB 8052 has recently been sequenced. Although transcriptome structure is important in order to reveal the functional and regulatory architecture of the genome, the physical structure of transcriptome for this strain, such as the operon linkages and transcript boundaries are not well understood.

Results: In this study, we conducted a single-nucleotide resolution analysis of the C. beijerinckii NCIMB 8052 transcriptome using high-throughput RNA-Seq technology. We identified the transcription start sites and operon structure throughout the genome. We confirmed the structure of important gene operons involved in metabolic pathways for acid and solvent production in C. beijerinckii 8052, including pta-ack, ptb-buk, hbd-etfA-etfB-crt (bcs) and ald-ctfA-ctfB-adc (sol) operons; we also defined important operons related to chemotaxis/motility, transcriptional regulation, stress response and fatty acids biosynthesis along with others. We discovered 20 previously non-annotated regions with significant transcriptional activities and 15 genes whose translation start codons were likely mis-annotated. As a consequence, the accuracy of existing genome annotation was significantly enhanced. Furthermore, we identified 78 putative silent genes and 177 putative housekeeping genes based on normalized transcription measurement with the sequence data. We also observed that more than $30 \%$ of pseudogenes had significant transcriptional activities during the fermentation process. Strong correlations exist between the expression values derived from RNA-Seq analysis and microarray data or qRT-PCR results.

Conclusions: Transcriptome structural profiling in this research provided important supplemental information on the accuracy of genome annotation, and revealed additional gene functions and regulation in C. beijerinckii.
\end{abstract}

\section{Background}

Solvents such as acetone, butanol and ethanol (ABE) produced through microbial fermentation represent important potential renewable fuels and chemicals [1]. Clostridium acetobutylicum and C. beijerinckii are among the prominent solvent-producing species. Although $C$. beijerinckii is phenotypically similar to $C$. acetobutylicum, the saccharolytic strains are phylogenetically distant from the amylolytic C. acetobutylicum ATCC 824 type strain [2]. C. beijerinckii exhibits a broader substrate range and optimum $\mathrm{pH}$ for growth and solvent production [3]; thus it may have

\footnotetext{
* Correspondence: blaschek@illinois.edu

${ }^{2}$ Institute for Genomic Biology, University of Illinois at Urbana-Champaign, Urbana, IL 61801, USA

Full list of author information is available at the end of the article
}

greater potential for biosolvent production than C. acetobutylicum.

The genome of C. beijerinckii NCIMB 8052 was sequenced by the DOE Joint Genome Institute in 2007 (JGI project ID 3634512). The genome size is $6.0 \mathrm{Mb}$, which is $50 \%$ larger than that of C. acetobutylicum ATCC 824. The C. beijerinckii 8052 solvent-producing genes are all located on the chromosome, as opposed to the location of these genes on a mega-plasmid in C. acetobutylicum 824. Although transcriptome structural organization is important in order to reveal the functional and regulatory architecture of the genome, such annotation for the C. beijerinckii 8052 genome is far from complete. Current genome annotation was made by computational analysis based on gene prediction algorithms. Although this allows for the determination of the complete set of gene loci and
Ciomed Central

() 2011 Wang et al; licensee BioMed Central Ltd. This is an Open Access article distributed under the terms of the Creative Commons Attribution License (http://creativecommons.org/licenses/by/2.0), which permits unrestricted use, distribution, and reproduction in any medium, provided the original work is properly cited. 
intergenic regions of the genome, it does not provide sufficient information concerning the transcriptional organization on a genome-wide level. For example, transcriptome structures such as operon linkages and transcript boundaries, etc. are not well understood and lack confirmation with experimental approaches. Next-generation highthroughput sequencing technology enabled us to obtain millions of cDNA reads simultaneously. In RNA-Seq analysis, these reads can be assembled against the genome sequence, and expression values calculated based on the reads mapped to genes. Deep sequencing of cDNA pool allowed us to study the bacterial transcriptome structure and gene expression at an unprecedented resolution and depth [4-8]. In this study, we used RNA-Seq technology to investigate $C$. beijerinckii 8052 transcriptome structure at a single-nucleotide resolution. We identified the transcription start sites and operon structure throughout the genome. We confirmed the structure of important gene operons involved in metabolic pathways for acid and solvent production in C. beijerinckii 8052. We defined important operons related to chemotaxis/motility, transcriptional regulation, stress response and fatty acids biosynthesis. We discovered 20 previously non-annotated regions with significant transcriptional activities and 15 genes whose translation start codons were likely misannotated. The results from this study significantly enhanced the accuracy of current genome annotation, and provided an essential reference point for other researchers working in related fields.

\section{Results and discussion}

\section{Growth kinetics and ABE fermentation}

C. beijerinckii 8052 grew rapidly with a very short lag phase in the batch fermentation with P2 medium supplemented with yeast extract and glucose (Figure 1A). The fermentation experienced a shift from acidogenesis to solventogenesis at approximately 4.5-8 h. Formation of solvents was detected at between 4.5-6.5 $\mathrm{h}$ after the start of fermentation, which corresponded to the late exponential growth phase. Butanol continued to increase throughout the stationary phase (Figure 1B). Samples for RNA isolation were collected at time points during acidogenesis (2 and $4.5 \mathrm{~h}$ ) and solventogenesis (after $6.5 \mathrm{~h}$ ) (Figure 1A). The combined information from these samples collected at different growth phases is representative for transcriptome structural analysis.

\section{Transcriptome definition and structure}

The 75-nt cDNA reads were mapped to the $C$. beijerinckii 8052 genome. Only those reads that mapped unambiguously to the genome were used for further analyses (Table 1). Collectively, around $75.5 \%$ of the C. beijerinckii 8052 genome was transcribed in at least one sample, even though the fraction in each single sample was much less.

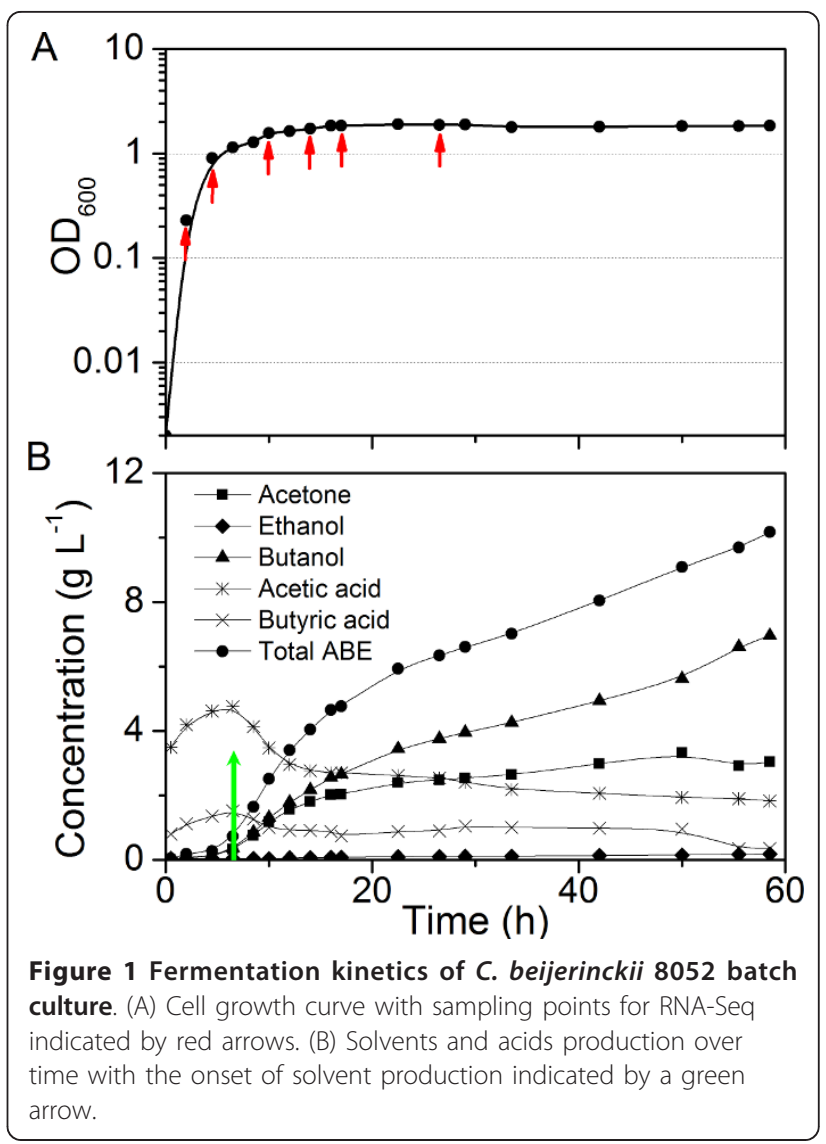

The sequence coverage per base was plotted and visualized using the genome browser Artemis and DNAPlotter $[5,9,10]$ (Additional file 1 Figure S1). The sequence coverage of the genes (based on number of mapped reads) for different samples was observed to be different in the overall sequence coverage profiles, which might be a reflection of the physiological states as the cell transited from one growth phase to another. For example, sample 2 represented a time period that was at the beginning of the transition from acidogenesis to solventogenesis. In this sample, genes encoding butyrate kinase (buk, 233080-234147 nt; Gene ID is listed in Additional file 2 Table S1, similarly hereinafter), acetyl-CoA acetyltransferase (thl,499121$500302 \mathrm{nt}$ ), glyceraldehyde-3-phosphate dehydrogenase (gap, 710763-711764 nt), fructose-bisphosphate aldolase (fba, 2199060-2199926 nt), aldehyde dehydrogenase (ald, 4399026-4400432 nt), acetoacetate decarboxylase (adc, 4401916-4402656 nt) were all actively expressed (high peaks in Additional file 1 Figure S1). These genes are involved in acid and butyryl-CoA formation, solventogenesis and glycolysis. The time frame of sample 4 was consistent with the transition to non-active growth and clostridial spores formation. In this sample, genes encoding stage $\mathrm{V}$ sporulation protein $\mathrm{T}$ (spoVT, 115288-115836 nt), spore coat protein cotJC ( $\operatorname{cotJC}, 2411617-2412183 \mathrm{nt})$, 
Table 1 Summary of RNA-Seq sequencing and data analysis results

\begin{tabular}{cccccccc}
\hline Sample & $\mathbf{1}$ & $\mathbf{2}$ & $\mathbf{3}$ & $\mathbf{4}$ & $\mathbf{5}$ & $\mathbf{6}^{*}$ & Total \\
\hline Time collected $(\mathrm{h})$ & 2 & 4.5 & 10 & 14 & 17 & 26.5 \\
Total No. of reads & 8988633 & 9457480 & 8011531 & 8448929 & 10363535 & 38574501 & 83844609 \\
No. of reads mapped & 8473125 & 9037616 & 7514804 & 7730815 & 9842491 & 37676913 & 80275764 \\
No. of reads unambiguously mapped & 6776544 & 7274568 & 6096405 & 6189652 & 8096169 & 35027722 & 69461060 \\
No. of bases unambiguously mapped & 508240800 & 545592600 & 457230375 & 464223900 & 607212675 & 2627079150 & 5209579500 \\
Percentage of genome represented & 38.71 & 36.59 & 43.84 & 40.60 & 41.34 & 50.67 & 75.46 \\
No. of genes with detectable expression** & 4219 & 4082 & 4496 & 4453 & 4487 & 4750 & 5024 \\
Range in expression levels (RPKM) & $3.2 \times 10^{-1}$ & $5.8 \times 10^{-2}$ & $4.5 \times 10^{-2}$ & $7.0 \times 10^{-2}$ & $1.0 \times 10^{-1}$ & $3.6 \times 10^{-2}$ & $3.6 \times 10^{-2}$ \\
& $\sim 2.5 \times 10^{4}$ & $\sim 6.0 \times 10^{4}$ & $\sim 2.5 \times 10^{4}$ & $\sim 9.0 \times 10^{4}$ & $\sim 8.6 \times 10^{4}$ & $\sim 9.8 \times 10^{4}$ & $\sim 9.8 \times 10^{4}$ \\
\hline
\end{tabular}

*Sample 6 was sequenced with 1 sample/lane, while samples 1-5 with 2 samples/lane. See Methods section for more details; **Pseudogenes included.

spore coat peptide assembly protein $\cot \mathrm{B}(\cot ) B, 2412190$ $2412453 \mathrm{nt})$, small acid-soluble spore protein, $\operatorname{sspA}(\operatorname{ssp} A$, 3596975-3597184 nt), small acid-soluble spore protein, sspC2 ( $s p C 2,3814296-3814505 \mathrm{nt})$ were actively expressed among others (Additional file 1 Figure S1). These genes are involved in the regulation of spore coat assembly and other sporulation-related processes $[11,12]$. These results were in good agreement with our previous observation using a 500-gene set DNA microarray [13]. A more detailed genome-wide transcriptional analysis of C. beijerinckii 8052 during the shift from acidogenesis to solventogenesis is currently underway in our lab.

The distinct transcript boundaries and multigene operon structure in a genome are essential for elucidating a genome's regulatory mechanisms. Through transcriptome profiling analysis, the transcript boundaries and operon structures were identified across the entire C. beijerinckii 8052 genome (Additional file 3 Table S2). Of all the $>5000$ genes, 2151 genes (42.2\%) were determined as being parts of multi-gene operon structures. The largest number of genes in a single operon was 32 (ribosomal proteins operon, Cbei_0150-0181, 192128207934 nt). A refined genome annotation file (in GenBank format) was generated (Additional file 4) based on the findings from this work and the current $C$. beijerinckii 8052 genome annotation in NCBI. The GenBank file can also be downloaded from https://netfiles.uiuc. edu/blaschek/www/Wang-BMC2011.

In addition, 5'-untranslated regions (5'-UTRs) were determined at the same time. Similar to other bacteria $[4,14]$, most of the identified 5'-UTRs were very short. Among all the 1605 5'-UTRs that were estimated with high confidence (that is, valid 5'-UTRs identified in the 6 samples with a $\leq 50 \mathrm{nt}$ window), 1262 were $\leq 50 \mathrm{nt}$, and only 65 were $\geq 100$ nt (Additional file 5 Table S3). RibEx [15] was used to test for the putative regulatory elements among the 5'-UTRs over $100 \mathrm{bp}$. Three Predicted Riboswitch-like Elements (do not belong to any Rfam [16]; yet present very significant associations with either a COG or a KEGG pathway) were found in the 5'-UTRs of
Cbei_0465, Cbei_1518 and Cbei_3542, respectively. In addition, a Known Riboswitch-like Element (flavin mononucleotide (FMN) riboswitch) was found in the 5'-UTR of Cbei_1224. More details about the identified putative riboswitches were described in Additional file 6 Table S4.

Previously, an operon containing genes encoding glyceraldehyde-3-phosphate dehydrogenase (gap), phosphoglycerate kinase $(p g k)$ and triosephosphate isomerase (tpi) was revealed by transcriptional analyses for $C$. acetobutylicum, while the gene encoding phosphoglycerate mutase $(\mathrm{pgm})$ was identified as not a member of this operon [17]. In this study, employing the single-nucleotide resolution sequence data, a similar gap-pgk-tpi operon was observed, upstream and apart from $p g m$ for C. beijerinckii 8052. In addition, it is very interesting that the upstream gene Cbei_0596 was also found to be linked with this operon (Figure 2A), which was validated by end-point RT-PCR as discussed below (Additional file 7 Figure S2 and Additional file 8 Table S5). The upstream gene (CA_C0708) of gap in C. acetobutylicum 824 genome is conjectured to be the counterpart of Cbei_0596 in C. beijerinckii 8052, since they both encode putative transcriptional regulator proteins and share $64 \%$ sequence identity. However, the intergenic distance between CA_C0708 and CA_C0709 in C. acetobutylicum 824 genome is $146 \mathrm{nt}$, while that between Cbei_0596 and Cbei_0597 in C. beijerinckii 8052 genome is only $71 \mathrm{nt}$. Although Cbei_0596-0599 are organized in the same operon, the sequence coverage depth downstream of gap was unexpectedly lower than that of the anterior (Figure 2A). An intrinsic terminator was predicted adjacent and downstream of gap (711818$711852 \mathrm{nt}$ ) using the bacterial genome transcription terminator prediction software TransTerm [18]. The terminator may have played a key role in attenuating the transcription of the downstream genes in this operon. Further experiments need to be carried out to confirm the activity and function of this element.

The butyryl-CoA formation related genes in C. acetobutylicum 824 encoding 3-hydroxybutyryl-CoA 


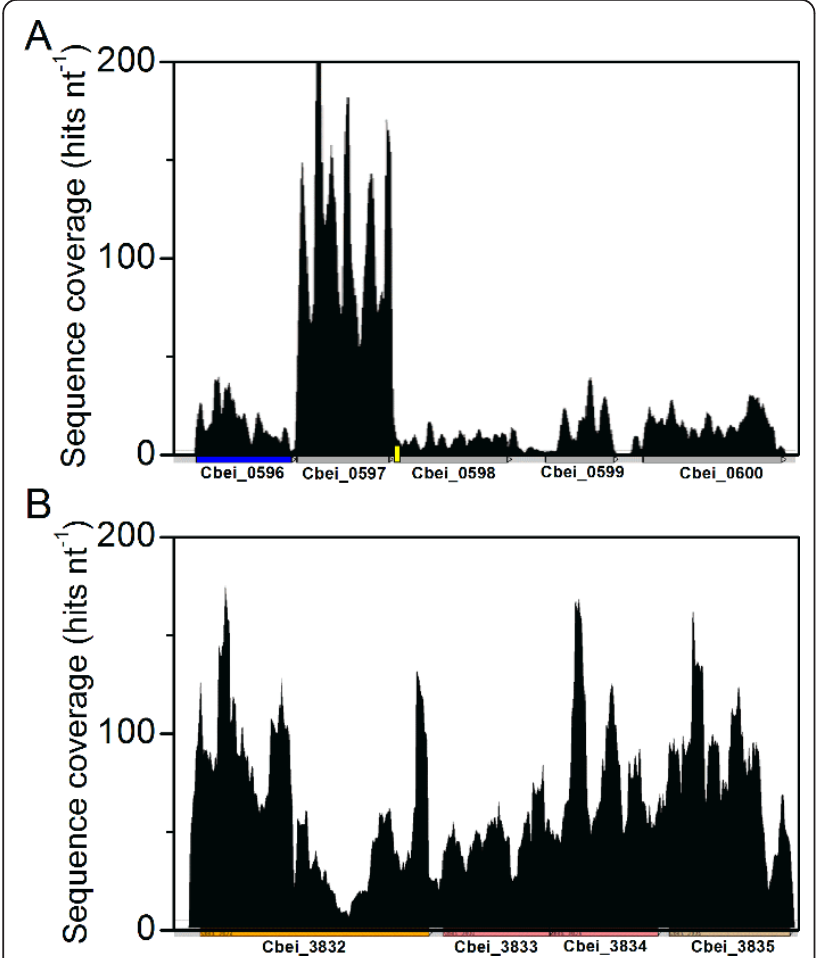

Figure 2 Single-nucleotide resolution-sequencing data revealed important operon structures in C. beijerinckii 8052. (A) The glycolytic genes (gap-pgk-tpi) operon structure (from sample 1). The upstream gene Cbei_0596 encoding a putative transcriptional regulator protein was also linked with this operon. The intrinsic terminator predicted with TransTerm [18] is represented with a small yellow bar. (B) The solventogenic genes are organized in the sol operon in the order of ald-ctfA-ctfB-adc. Gene positions and directions are shown beneath by arrows. The color of arrows indicates the COG functional class of genes as defined in Additional file 1 Figure S1.

dehydrogenase $(h b d)$, crotonase $(c r t)$, and butyryl-CoA dehydrogenase $(b c d)$ were identified as a $b c s$ (butyrylCoA synthesis) operon in the order of $c r t$-bcd-etfB (encoding electron transfer flavoprotein subunit beta)etfA (encoding electron transfer flavoprotein subunit alpha)-hbd [19,20]. A homologous operon structure was observed for C. beijerinckii 8052 (Cbei_0321-0325) transcribed in the same order but the opposite direction (Additional file 3 Table S2). In addition, based on the sequencing data, similar $p f k$ (phosphofructokinase)pyk (pyruvate kinase), pta (phosphotransacetylase)-ack (acetate kinase), $p t b$ (phosphate butyryltransferase)-buk gene operons were identified in C. beijerinckii 8052 as those described in C. acetobutylicum 824 [21-23] (Additional file 3 Table S2). Among them, $p t b-b u k$ operon was also verified by end-point RT-PCR experiment as discussed below (Additional file 7 Figure S2 and Additional file 8 Table S5).

Nearly all the genes encoding solventogenic enzymes have been cloned and characterized in C. acetobutylicum
[24]. In C. acetobutylicum 824, the genes adhE (acetaldehyde-CoA/alcohol dehydrogenase), ctfA (acetoacetylCoA: acetate/butyrate-CoA transferase subunit A) and ctfB (acetoacetyl-CoA: acetate/butyrate-CoA transferase subunit B) are located in the sol (solvent formation) operon on the mega-plasmid pSOL1 whose loss leads to the degeneration of the strain [25], while adc (acetoacetate decarboxylase) is organized in a monocistronic operon in the opposite direction [26,27]. In this study, a sol operon organized in the order of ald-ctfA-ctfB-adc was observed (Figure 2B and Additional file 3 Table S2). Previously, Chen and Blaschek (1999) speculated that the ald, $c t f A, c t f B$ and $a d c$ genes were located in an operon following a Northern hybridization analysis of C. beijerinckii 8052 total RNA [28]. With direct sequencing data, this study successfully confirmed the above hypothesis, and identified the transcriptional start sites (TSS) upstream of ald gene. This solventogenic gene arrangement in C. beijerinckii 8052 is consistent with that observed in C. beijerinckii NRRL B593 and C. saccharoperbutylacetonicum N1-4 [29,30].

A flagellar/chemotaxis gene operon (CA_C2225-C2215) was previously defined in C. acetobutylicum [31]; a counterpart organized in exactly the same order (Cbei_43124302) in C. beijerinckii 8052 was observed in this study (Additional file 3 Table S2). Similarly to C. acetobutylicum 824 , the transcriptional regulator sigF operon was also confirmed in C. beijerinckii 8052 with the sequencing data that includes the forespore-specific sigma factor gene sigF, the anti-sigF factor gene spoIIAB, and the anti-anti-sigF factor gene spoIIAA (Additional file 3 Table S2). In Bacillus subtilis and C. acetobutylicum, the class I heat shock genes are organized in dnaKJ (organized in the order of hrcA-grpE-dnaK-dnaJ in C. acetobutylicum 824) and groESL (groES-groEL) operons [32,33]. The similar organization of dnaKJ and groESL operons for C. beijerinckii 8052 was also observed in this study (Additional file 3 Table S2). The fatty acid biosynthesis genes are organized in a single $f a b$ operon in C. acetobutylicum (CA_C3568C3580) [34], while in this study, the fab genes (Cbei_10671077) in C. beijerinckii 8052 were observed to be organized in four operons, which is similar to those of B. subtilis [35] (Additional file 3 Table S2).

While the current $C$. beijerinckii 8052 genome was annotated based on bioinformatical predictions, the RNASeq sequencing approach provides additional experimental evidence for genome annotation. By comparing the sequence coverage data to the genome annotation, 20 non-annotated regions were found to have significant transcriptional activities (Figure 3A, see also the supplemental texts in Additional file 9). These regions may represent potential new genes or regulatory RNAs. Twelve potential new genes were predicted in these regions using GeneMark [36]. Additional details about this test and the 


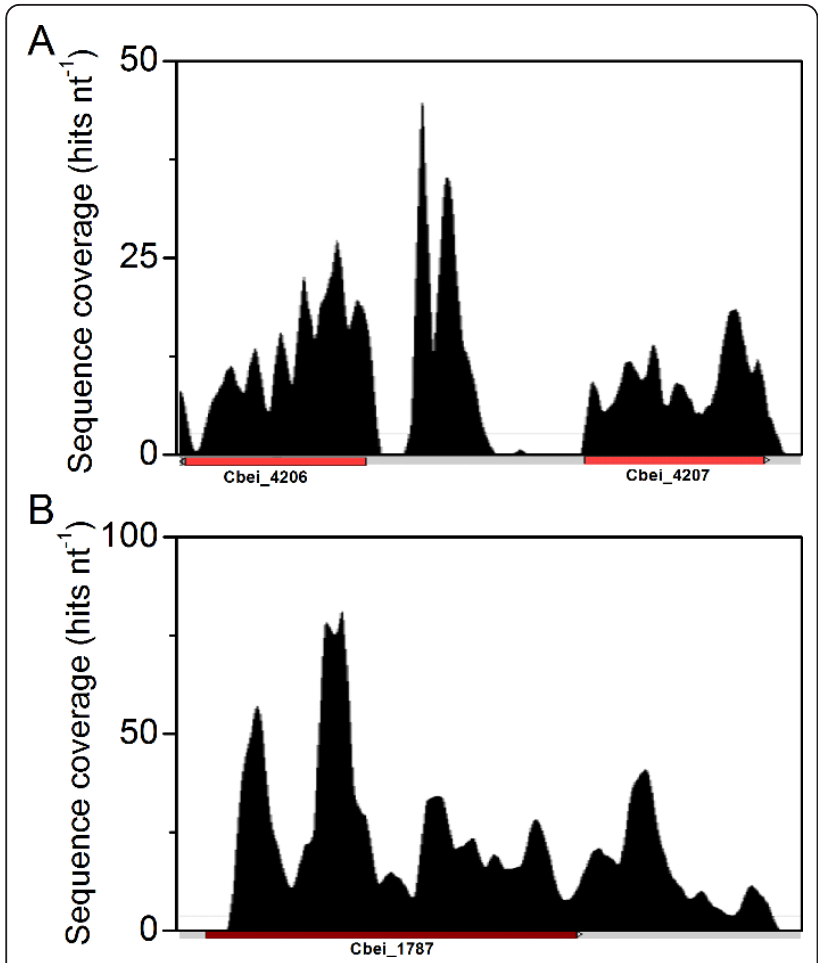

Figure 3 Mapping the high resolution RNA-Seq data back to the genome allows the test of current $C$. beijerinckii 8052 genome annotation. (A) Sequence coverage in the Cbei_42064207 region, showing transcription activity across a region not included in the current genome annotation (from sample 2). (B) Sequence coverage near Cbei_1787, with the sequence coverage starts downstream the annotated translation start codon (from sample 5). Gene positions and directions are shown beneath by arrows. The color of arrows indicates the COG functional class of genes as defined in Additional file 1 Figure S1.

predicted genes were summarized in Additional file 10 Table S6.

In addition, 15 transcripts were identified with TSS that are downstream of the current annotated translation start sites (Figure 3B, see also the supplemental texts in Additional file 9). For these regions, re-annotation is needed since the current start codons may have been mis-annotated.

\section{Putative silent genes}

Based on RNA-Seq sequence data, 78 protein-encoding genes demonstrated no transcripts over all six sampling time points, and these genes are likely silent (Additional file 11 Table S7). Thirty-one out of them were genes encoding hypothetical proteins. In addition, half of the genes encoding transposases (14 out of the total 29 in the genome) were silent. Although transposases may have played important roles during the evolution of C. beijerinckii, most of them are not as functional any more during the course of a batch fermentation. Besides, several genes encoding the subunits (such as soborsespecific subunits, lactose/cellobiose-specific subunits) of phosphotransferase system (PTS) related to the transport of sugars other than glucose were also among the list of silent genes. Since glucose was the only carbohydrate used in this study, these enzymes were not induced during the fermentation process.

\section{Putative housekeeping genes (HKGs)}

Some genes have little variation in expression level through the entire fermentation process, and they are regarded as putative housekeeping genes (HKGs). For accurate gene expression quantification, normalization of gene expression against HKGs (endogenous control or reference gene) is generally required. In this study, 177 protein-coding genes were identified as putative HKGs with the lowest coefficient of variation (CV) in RPKM (see Methods section) values among all the sampling points $(\mathrm{CV}=$ standard deviation/mean; $<30 \%$ for listed genes in Additional file 12 Table S8) [37]. A COG functional group analysis by Fisher's exact test found that COG functional category D (Cell cycle control, mitosis and meiosis, 10.3\%), L (Replication, recombination and repair, 9.2\%) and $\mathrm{E}$ (Amino acid transport and metabolism, 6.6\%) were overrepresented in this list $[37,38]$. This list of putative HKGs was generated based on the transcription data obtained from the six samples under the certain batch fermentation conditions employed in this study. This list can be considered as a starting point for identifying HKGs for C. beijerinckii. However, whether these genes are stably expressed under different experimental conditions requires further study.

A prediction of the promoters for primary sigma factors for all the putative HKGs was carried out using BPROM (http://linux1.softberry.com/berry.phtml). In total, 88 promoters along with their corresponding -10 box and -35 box sequences were predicted (Additional file 12 Table S8).

\section{Pseudogenes}

Pseudogenes in bacteria genomes are non-functional copies of gene fragments usually created by random mutations and chance events $[39,40]$. Although pseudogenes are usually non-functional, it has been reported that quite a few of them can still go through the transcription process [41-43]. In this study, 26 out of the 82 pseudogenes in C. beijerinckii 8052 genome were found to have significant transcriptional activities over the course of fermentation (Additional file 13 Table S9). However, although globally the 82 pseudogenes comprise about $0.6 \%$ of the predicted CDS of the genome, only < $0.1 \%$ of all the RNA-Seq reads mapped to these genes, 
indicating a significantly lower transcriptional activity when compared to the regular protein coding genes. In addition, six pseudogenes were found to be completely silent throughout the fermentation process (Additional file 13 Table S9). This is overrepresented among the silent genes when compared to the protein-coding genes based on a Fisher's exact test $(p=0.0015)$.

When pseudogenes share high sequence identity with other functional genes in the genome, it is usually on one hand very difficult to design probes to detect the transcription of pseudogenes with traditional methods, and on the other pseudogenes can lead to amplification bias during genetic studies of the functional genes with high sequence identity to pseudogenes [42]. Apparently such problems can be avoided with RNA-Seq method, which is one of the unprecedented advantages of RNASeq technique.

For summarization and easy reference to the readers, a table (Additional file 14 Table S10) summarizing the various supplementary information provided by RNA-Seq study to the current genome annotation was listed in the supplemental materials.

\section{End-point RT-PCR}

End-point RT-PCR was used to validate the operon structure results obtained from RNA-Seq analysis [4]. One gene pair that is highly likely to be co-operonic with an intergenic distance of $7 \mathrm{bp}$ was chosen as a positive control (Cbei_0341-0342). Ten other gene pairs with long intergenic distances (71-440 bp) where there were high transcription levels were chosen. All the operon structures determined by sequence data in the chosen gene pairs were confirmed by end-point RT-PCR results, indicating that RNA-Seq is a valid approach for operon structure identification (Additional file 7 Figure S2 and Additional file 8 Table S5). Among them, the $p t b$-buk operon and the linkage between Cbei_0596 and Cbei_0597 (gap) as discussed above were also confirmed.

\section{Correlations of RNA-Seq data with microarray and qRT- PCR data}

The RNA-Seq data obtained in this study were compared to the results obtained previously using a 500 -gene set DNA microarray [13]. A small number of genes that did not allow for unambiguous mapping in RNA-Seq analysis were not included in the comparison. A good correlation was observed between the normalized coverage depth for sample 2 (collected at $4.5 \mathrm{~h}$ ) and the raw microarray fluorescence intensities for an equivalent sample (collected at $5 \mathrm{~h}$ under the same growth condition) (Figure 4A). Specifically, the gene expression patterns of glycolytic genes Cbei_0597-0600 and sporulation genes Cbei_2069-2071 from samples $2(4.5 \mathrm{~h})$ and $4(14 \mathrm{~h})$ measured by

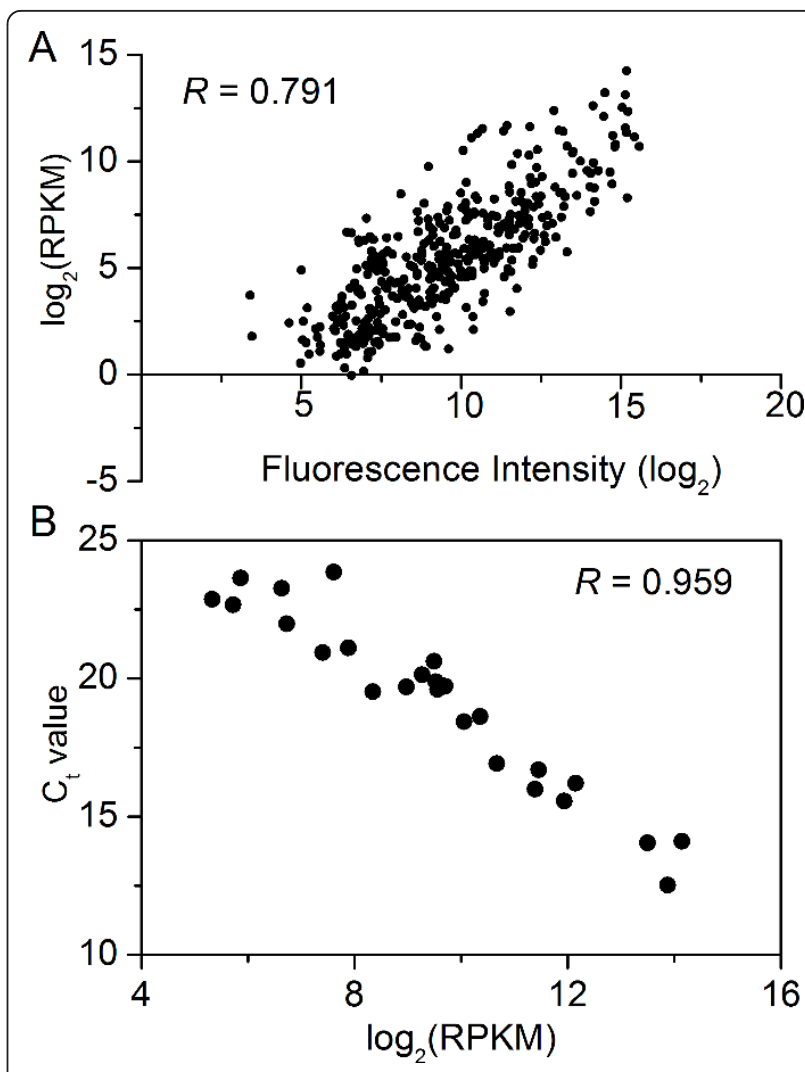

Figure 4 Correlations of RNA-Seq data with microarray and qRTPCR data. (A) Comparison of normalized sequence coverage depth from RNA-Seq and absolute microarray fluorescence intensity. Shown is a plot of $\log _{2}$-transformation of RPKM for sample 2 (at $4.5 \mathrm{~h}$ ) and raw microarray intensities for an equivalent sample (at 5 h) from Shi and Blaschek [13]. (B) Real time qRT-PCR verification of RNA-Seq results for selected genes (from sample 4, see also Additional file 15 Table S11).

sequencing data were compared. Similar differential expression patterns by microarray data from equivalent samples (5 h vs. $13 \mathrm{~h}$ ) were observed (Figure 5). Although only a limited number of genes were compared, the good correlation between two methods further demonstrated the effectiveness of RNA-Seq approach. An additional advantage of RNA-Seq is that the sequence data can measure expression levels for every gene without bias caused by sequence-specific differences in hybridization efficiency in microarray-based methods $[4,44]$, and sequence data allow one to measure gene expression more accurately with a much higher dynamic range as indicated in this study (Table 1 ) and other references $[4,8,44,45]$.

The expression measurement with RNA-Seq data was further validated using real time quantitative reverse transcription PCR (qRT-PCR). Twenty-five genes were selected for the test (Additional file 13 Table S9). A high degree of correlation $(R=0.959)$ between the threshold value $(\mathrm{Ct})$ and the $\log _{2}$-tranformation of RPKM was observed (Figure 4B). 


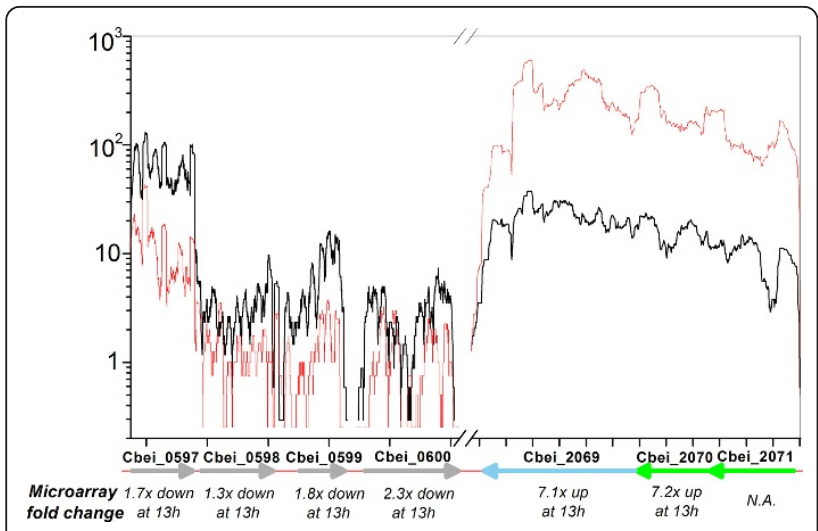

Figure 5 Comparison of transcriptional profiles for sample 2 (4.5 $\mathrm{h}$, black line) and sample 4 ( $14 \mathrm{~h}$, red line), normalized to the gene length and genome-wide total number of unambiguously mapped reads for that sample. Shown are an $\sim 5.4 \mathrm{~kb}$ segment surrounding the gap-pgk-tpi glycolytic gene operon (Cbei_0597-0600) and an $\sim 1.2$ kb segment surrounding the cotJC-cotJB (Cbei_2069-2071) sporulation gene operon. Specific genes are indicated with arrows, whose colors indicate the COG functional class of genes as defined in Additional file 1 Figure S1. Below each gene is the expression level fold change that was measured by microarray data for equivalent samples (5 h vs. 13 h in Shi and Blaschek [13]; microarray data for Cbei_2071 are not available).

\section{Conclusions}

A single-nucleotide resolution analysis of the $C$. beijerinckii NCIMB 8052 transcriptome structure was conducted using high-throughput RNA-Seq technology. The transcription start sites and operon structures were identified throughout the genome. The structure of operons involved in metabolic pathways for acid and solvent production in C. beijerinckii 8052 were confirmed. Important operons related to chemotaxis/motility, transcriptional regulation, stress response and fatty acids biosynthesis along with others were defined. Twenty previously non-annotated regions were discovered with significant transcriptional activities and 15 genes were identified whose translation start codons were likely mis-annotated. As a consequence, the accuracy of existing genome annotation was significantly enhanced. Moreover, 78 silent genes and 177 putative housekeeping genes were identified based on normalized transcription measurement with the sequence data. More than $30 \%$ of the pseudogenes were observed to have significant transcriptional activities during the fermentation process. Strong correlations exist between the expression values derived from RNA-Seq analysis and microarray data or qRT-PCR results. Transcriptome structural profiling in this study provided important supplemental information on the accuracy of annotation of the $C$. beijerinckii genome.

\section{Methods}

\section{Bacterial culture and fermentation experiment}

Laboratory stocks of C. beijerinckii 8052 spores were stored in sterile $\mathrm{H}_{2} \mathrm{O}$ at $4{ }^{\circ} \mathrm{C}$ [46]. Spores were heatshocked at $80^{\circ} \mathrm{C}$ for $10 \mathrm{~min}$, followed by cooling on ice for $5 \mathrm{~min}$. The heat-shocked spores were inoculated into tryptone-glucose-yeast extract (TGY) medium containing $30 \mathrm{~g} \mathrm{~L}^{-1}$ tryptone, $20 \mathrm{~g} \mathrm{~L}^{-1}$ glucose, $10 \mathrm{~g} \mathrm{~L}^{-1}$ yeast extract and $1 \mathrm{~g} \mathrm{~L}^{-1} \mathrm{~L}$-cysteine at a $1 \%$ inoculum level. The TGY culture was incubated at $35 \pm 1{ }^{\circ} \mathrm{C}$ for $12-14 \mathrm{~h}$ in an anaerobic chamber under $\mathrm{N}_{2}: \mathrm{CO}_{2}: \mathrm{H}_{2}$ (volume ratio of $85: 10: 5$ ) atmosphere. Subsequently, actively growing culture was inoculated into a model solution containing $60 \mathrm{~g} \mathrm{~L}^{-1}$ glucose, $1 \mathrm{~g} \mathrm{~L}^{-1}$ yeast extract, and filter-sterilized P2 medium $[47,48]$ in a Sixfors bioreactor system (Infors AG, Bottmingen, Switzerland). Throughout the experiment, oxygen-free nitrogen was flushed through the broth to maintain anaerobiosis. Temperature was controlled at $35 \pm 1^{\circ} \mathrm{C}$. A stirring at $50 \mathrm{rpm}$ was employed for mixing. During the course of fermentation, samples were collected for cell density and product concentration measurements. For sequencing purpose, RNA samples were taken over the early exponential, late exponential and stationary phases (sample $1-6$ at 2, 4.5, 10, 14, 17 and 26.5 h respectively as shown in Figure 1A).

\section{Culture growth and fermentation products analysis}

Culture growth was measured by following optical density (OD) in the fermentation broth at $\mathrm{A}_{600}$ using a BioMate 5 UV-Vis Spectrophotometer (Thermo Fisher Scientific Inc., Waltham, MA). ABE, acetic acid, and butyric acid concentrations were quantified using gas chromatography (GC) system as previously described [49].

\section{RNA isolation, library construction and sequencing}

In preparation for RNA isolation, $10 \mathrm{ml}$ cultures were harvested at six time points, and centrifuged at 4,000 $\times \mathrm{g}$ for $10 \mathrm{~min}$ at $4^{\circ} \mathrm{C}$. Total RNA was extracted from the cell pellet using Trizol reagent based on manufacture's protocol (Invitrogen, Carlsbad, CA) and further purified using RNeasy minikit (Qiagen, Valencia, CA). DNA was removed using a DNA-free ${ }^{\mathrm{TM}}$ kit (Ambion Inc., Austin, TX). RNA quality was assessed using a nanochip on a model 2100 bioanalyzer (Agilent Technologies, Santa Clara, CA). RNA concentration was determined with a nanodrop (Biotek Instruments, Winooski, VT). Bacterial $16 \mathrm{~S}$ and $23 \mathrm{~S}$ ribosomal RNAs were removed with a MICROBExpress ${ }^{\mathrm{TM}}$ kit (Ambion Inc., Austin, TX). The enriched mRNA was converted to a RNA-Seq library using the mRNA-Seq library construction kit (Illumina Inc., San Diego, CA) following manufacturer's protocols. For samples 1 to 6 , two samples were pooled and 
sequenced on one single lane of an eight-lane flow cell with the Genome Analyzer IIx system (Illumina Inc., San Diego, CA). However, sample 6 yielded a poor read quality following the first sequencing. In order to obtain enough sequencing depth, sample 6 was sequenced again using one single lane under otherwise identical conditions. The derived sequence reads were $75 \mathrm{nt}$ long. The overall error rate of the control DNA was $<0.6 \%$. The total number of reads generated from each library is summarized in Table 1.

\section{Sequence mapping and visualization}

The generated 75-nt reads were mapped to the $C$. beijerinckii 8052 genome using MAQ, and those that did not align uniquely to the genome were discarded $[5,50]$. The quality parameter (-q) used in MAQ pileup was set to 30 . Each base was assigned a value based on the number of mapped sequence coverage. The coverage plot files were read into Artemis and visualized as sequence coverage profiles over the entire genome [5,9]. Transcription start sites (TSS) were manually identified as described in Passalacqua et al. [4]. The region between determined TSS and the annotated translation start site was defined as the 5'-untranslated region (5'-UTR).

\section{Measurement of gene expression}

The quantitative gene expression value, RPKM (reads/Kb/ Million), was calculated using custom Perl scripts by normalizing the sequence coverage over the gene length and total unambiguously mapped reads in each library [7,37].

\section{End-point RT-PCR for operon structure assessment}

End-point RT-PCR was performed as described in Passalacqua et al. [4]. For each selected co-operonic gene pair, four primers $1 \mathrm{~F}, 1 \mathrm{R}, 2 \mathrm{~F}$ and $2 \mathrm{R}$ were designed. Primers $1 \mathrm{~F}$ and $1 \mathrm{R}$ amplify a region within gene 1 , while $2 \mathrm{~F}$ and $2 \mathrm{R}$ amplify a region within gene 2 . When a continuous transcript exists, $1 \mathrm{~F}$ and $2 \mathrm{R}$ amplify across the intergenic region between genes 1 and 2 . Therefore, the size of the end-point RT-PCR product will be the same using either cDNA or genomic DNA as templates. The reaction products were visualized on $1.5 \%$ agarose gels stained with ethidium bromide.

\section{Real time qRT-PCR}

Quantitative reverse transcription PCR (qRT-PCR) was performed in order to validate the quantification of gene expression level by RNA-Seq. Twenty-five genes were chosen to represent a large range of RPKM values (from $\sim 40$ to $>18000$ ). Triplicate reactions were performed using Power SYBR green PCR master mix (Applied Biosystems, Carlsbad, CA) on an ABI Prism 7900 HT fast real-time PCR machine (Applied Biosystesms). Detected genes and primer sequences are listed in Additional file 15 Table S11.

\section{RNA-Seq data accession number}

The RNA-Seq sequencing data have been deposited in the NCBI Sequence Read Archive (SRA) under the accession number SRA045799.

\section{Additional material}

Additional file 1: Circular plots of the reads from all six samples mapping to the $C$. beijerinckii $\mathbf{8 0 5 2}$ genome. The outermost and second outermost circles represent CDS on the forward and reverse strands respectively, both of which are colored according to Clusters of Orthologous Groups (COG) functional classification assigned to $C$. beijerinckii 8052 annotation. The gold peak and shading area represents greater than the average and lower (in purple). The COG functional classes and corresponding color-coding are as follows (with RGB color model values in the parentheses): Class J, black ( 000$)$; Class K, blue $\left(\begin{array}{ll}0 & 0\end{array}\right.$ 255); Class L, brown (165 42 42); Class B, dark blue (0 0 139); Class D, chocolate (210 105 30); Class V, cyan (0 255 255); Class T, red (255 0 0); Class M, yellow (255 255 0); Class N, dark green (0 100 0); Class U, grey (128 128 128); Class O, gold (255 215 0); Class C, orange (255 165 0); Class G, light grey (170 170 170); Class E, mid red (255 63 63); Class F, pink (255 192 203); Class H, purple (128 0 128); Class I, violet (238 130 138); Class P, skyblue (135 206 235); Class Q, $\tan (210180$ 140); Class R, darkgrey (100 100 100); Class S, darkred (139 0 0); Not in COGs, green (0 255 0). If one gene belongs to more than one COG classes, the color for that gene was defined by the first class it belongs to as the above order.

Additional file 2: The corresponding Gene ID and ortholog gene in C. acetobutylicum ATCC 824 for the genes in C. beijerinckii 8052 mentioned in the paper (three-letter short names were used in the main text).

Additional file 3: TSS and operon structure in C. beijerinckii 8052 chromosome.

Additional file 4: Refined genome annotation (in GenBank format) based on the findings from this work and the current $C$. beijerinckii $\mathbf{8 0 5 2}$ genome annotation in NCBI. The GenBank file can also be downloaded from https://netfiles.uiuc.edu/blaschek/www/WangBMC2011.

Additional file 5: Genes with 5'-UTR length $\geq 100 \mathrm{nt}$. Additional file 6: Putative riboswitches identified using RibEx among the 5'-UTRs over $100 \mathrm{nt}$.

Additional file 7: Images of end-point RT-PCR products for 11 selected co-operonic gene pairs on a $1.5 \%$ agarose gel. For each gene pair (1-11, see details in Additional file 8, Table S5) as labeled on the top, the band on the left lane is PCR product using CDNA reversetranscribed from RNA samples as template, on the right is PCR product using genomic DNA as template.

Additional file 8: Operon structure validation with end-point RTPCR.

Additional file 9: Supplemental texts.

Additional file 10: Potential new genes predicted in non-annotated regions with significant transcriptional activities using GeneMark. Additional file 11: Putative silent genes over all the six sampling time points

Additional file 12: Putative housekeeping genes (HKGs)

Additional file 13: Transcription of the pseudogenes.

Additional file 14: Summary of the supplementary information provided by RNA-Seq to the current C. beijerinckii 8052 genome annotation

Additional file 15: Genes and primer sequences for qRT-PCR test 


\section{Acknowledgements}

This work was supported by USDA Research Initiative Award AG 2006-3550417419, and Illinois Council on Food and Agricultural Research (C-FAR) Grant 698 IDA CF06DS-01-03 to HPB. We thank Dr. Heng Li and Dr. Timothy Perkins for their helpful inputs on the data analysis.

\section{Author details}

'Department of Agricultural and Biological Engineering, University of Illinois at Urbana-Champaign, Urbana, IL 61801, USA. ${ }^{2}$ Institute for Genomic Biology, University of Illinois at Urbana-Champaign, Urbana, IL 61801, USA. ${ }^{3}$ Department of Animal Sciences, University of Illinois at Urbana-Champaign, Urbana, IL 61801, USA. ${ }^{4}$ Department of Food Science and Human Nutrition, University of Illinois at Urbana-Champaign, Urbana, IL 61801, USA. ${ }^{5}$ Center for Advanced Bioenergy Research (CABER), University of Illinois at UrbanaChampaign, Urbana, IL 61801, USA

\section{Authors' contributions}

YW, XL, HPB conceived and designed the study. YW and XL performed the experiments. YW, $X L$ and $Y M$ analyzed the RNA-Seq data. YW, $X L$ and HPB wrote the manuscript, with input from all authors. All authors discussed the results, read and approved the final manuscript.

\section{Competing interests}

The authors declare that they have no competing interests.

Received: 2 June 2011 Accepted: 30 September 2011

Published: 30 September 2011

\section{References}

1. Wu M, Wang M, Liu JH, Huo H: Assessment of potential life-cycle energy and greenhouse gas emission effects from using corn-based butanol as a transportation fuel. Biotechnol Progr 2008, 24(6):1204-1214.

2. Keis S, Bennett CF, Ward VK, Jones DT: Taxonomy and phylogeny of industrial solvent-producing clostridia. Int J Syst Bacteriol 1995, 45(4):693-705.

3. Ezeji T, Blaschek HP: Fermentation of dried distillers' grains and solubles (DDGS) hydrolysates to solvents and value-added products by solventogenic clostridia. Bioresource Technol 2008, 99(12):5232-5242.

4. Passalacqua KD, Varadarajan A, Ondov BD, Okou DT, Zwick ME, Bergman NH: Structure and complexity of a bacterial transcriptome. $J$ Bacteriol 2009, 191(10):3203-3211.

5. Perkins $T$, Kingsley RA, Fookes MC, Gardner PP, James KD, Yu L, Assefa SA, He M, Croucher NJ, Pickard DJ, et al: A strand-specific RNA-Seq analysis of the transcriptome of the typhoid bacillus Salmonella typhi. PLoS Genet 2009, 5(7).

6. Wurtzel O, Sapra R, Chen F, Zhu YW, Simmons BA, Sorek R: A single-base resolution map of an archaeal transcriptome. Genome Res 2010, 20(1):133-141.

7. Mortazavi A, Williams BA, McCue K, Schaeffer L, Wold B: Mapping and quantifying mammalian transcriptomes by RNA-Seq. Nat Methods 2008, 5(7):621-628.

8. Nagalakshmi U, Wang Z, Waern K, Shou C, Raha D, Gerstein M, Snyder M: The transcriptional landscape of the yeast genome defined by RNA sequencing. Science 2008, 320:1344-1349.

9. Carver T, Berriman M, Tivey A, Patel C, Bohme U, Barrell BG, Parkhill J, Rajandream M-A: Artemis and ACT: viewing, annotating and comparing sequences stored in a relational database. Bioinformatics 2008 24(23):2672-2676.

10. Carver T, Thomson N, Bleasby A, Berriman M, Parkhill J: DNAPlotter: circular and linear interactive genome visualization. Bioinformatics 2009, 25(1):119-120.

11. Steil L, Serrano M, Henriques AO, Volker U: Genome-wide analysis of temporally regulated and compartment-specific gene expression in sporulating cells of Bacillus subtilis. Microbiology-(UK) 2005, 151:399-420

12. Wang ST, Setlow B, Conlon EM, Lyon JL, Imamura D, Sato T, Setlow $P$, Losick $R$, Eichenberger $P$ : The forespore line of gene expression in Bacillus subtilis. J Mol Biol 2006, 358(1):16-37.

13. Shi Z, Blaschek HP: Transcriptional analysis of Clostridium beijerinckii NCIMB 8052 and the hyper-butanol-producing mutant BA101 during the shift from acidogenesis to solventogenesis. Appl Environ Microb 2008, 74(24):7709-7714.
14. McGrath PT, Lee H, Zhang L, Iniesta AA, Hottes AK, Tan MH, Hillson NJ, Hu P, Shapiro L, McAdams HH: High-throughput identification of transcription start sites, conserved promoter motifs and predicted regulons. Nat Biotechnol 2007, 25(5):584-592.

15. Abreu-Goodger C, Merino E: RibEx: a web server for locating riboswitches and other conserved bacterial regulatory elements. Nucleic Acids Res 2005, 33:W690-W692.

16. Griffiths-Jones S, Moxon S, Marshall M, Khanna A, Eddy SR, Bateman A: Rfam: annotating non-coding RNAs in complete genomes. Nucleic Acids Res 2005, 33:D121-D124.

17. Schreiber W, Dürre P: Differential expression of genes within the gap operon of Clostridium acetobutylicum. Anaerobe 2000, 6(5):291-297.

18. Ermolaeva MD, Khalak HG, White O, Smith HO, Salzberg SL: Prediction of transcription terminators in bacterial genomes. J Mol Biol 2000, 301 (1):27-33.

19. Bennett GN, Rudolph FB: The central metabolic pathway from acetyl-CoA to butyryl-CoA in Clostridium acetobutylicum. Fems Microbiol Rev 1995, 17(3):241-249.

20. Boynton ZL, Bennett GN, Rudolph FB: Cloning, sequencing, and expression of clustered genes encoding $\beta$-hydroxybutyryl-coenzyme $A$ (CoA) dehydrogenase, crotonase, and butyryl-CoA dehydrogenase from Clostridium acetobutylicum ATCC 824. J Bacteriol 1996, 178(11):3015-3024.

21. Belouski E, Watson DE, Bennett GN: Cloning, sequence, and expression of the phosphofructokinase gene of Clostridium acetobutylicum ATCC 824 in Escherichia coli. Curr Microbiol 1998, 37(1):17-22.

22. Boynton ZL, Bennett GN, Rudolph FB: Cloning, sequencing, and expression of genes encoding phosphotransacetylase and acetate kinase from Clostridium acetobutylicum ATCC 824. Appl Environ Microb 1996, 62(8):2758-2766.

23. Walter KA, Nair RV, Cary JW, Bennett GN, Papoutsakis ET: Sequence and arrangement of two genes of the butyrate-synthesis pathway of Clostridium acetobutylicum ATCC 824. Gene 1993, 134(1):107-111.

24. Düerre P: Formation of solvents in clostridia. In Handbook on clostridia. Edited by: Düerre P. London: CRC press; 2005:671-693.

25. Cornillot E, Nair RV, Papoutsakis ET, Soucaille P: The genes for butanol and acetone formation in Clostridium acetobutylicum ATCC 824 reside on a large plasmid whose loss leads to degeneration of the strain. $J$ Bacteriol 1997, 179(17):5442-5447.

26. Gerischer U, Dürre P: Cloning, sequencing, and molecular analysis of the acetoacetate decarboxylase gene region from Clostridium acetobutylicum. J Bacteriol 1990, 172(12):6907-6918.

27. Petersen DJ, Cary JW, Vanderleyden J, Bennett GN: Sequence and arrangement of genes encoding enzymes of the acetone-production pathway of Clostridium acetobutylicum ATCC 824. Gene 1993, 123(1):93-97.

28. Chen CK, Blaschek HP: Effect of acetate on molecular and physiological aspects of Clostridium beijerinckii NCIMB 8052 solvent production and strain degeneration. Appl Environ Microb 1999, 65(2):499-505.

29. Rui H: The cloning of a putative regulatory gene and the sol region from Clostridium beijerinckii. Master thesis Virginia Polytechnic Institute and State University, Department of Biochemistry; 1999.

30. Kosaka T, Nakayama S, Nakaya K, Yoshino S, Furukawa K: Characterization of the sol operon in butanol-hyperproducing Clostridium saccharoperbutylacetonicum strain N1-4 and its degeneration mechanism. Biosci Biotechnol Biochem 2007, 71(1):58-68.

31. Paredes CJ, Rigoutsos I, Papoutsakis ET: Transcriptional organization of the Clostridium acetobutylicum genome. Nucleic Acids Res 2004, 32(6):1973-1981

32. Bahl H, Muller H, Behrens S, Joseph H, Narberhaus F: Expression of heat shock genes in Clostridium acetobutylicum. Fems Microbiol Rev 1995, 17(3):341-348.

33. Tomas CA, Welker NE, Papoutsakis ET: Overexpression of groESL in Clostridium acetobutylicum results in increased solvent production and tolerance, prolonged metabolism, and changes in the cell's transcriptional program. Appl Environ Microb 2003, 69(8):4951-4965.

34. Tomas CA, Beamish J, Papoutsakis ET: Transcriptional analysis of butanol stress and tolerance in Clostridium acetobutylicum. J Bacteriol 2004, 186(7):2006-2018.

35. de Mendoza D, Schujman GE, Aguilar PS: Biosynthesis and function of membrane lipids. In Bacillus subtilis and its closest relatives: from genes to cells. Edited by: Sonenshein A, Hoch J, Losick R. Washington, D.C.: ASM Press; 2002:43-55. 
36. Besemer J, Borodovsky M: GeneMark: web software for gene finding in prokaryotes, eukaryotes and viruses. Nucleic Acids Res 2005, 33(Suppl 2): W451-W454.

37. Severin A, Woody J, Bolon Y-T, Joseph B, Diers B, Farmer A, Muehlbauer G, Nelson R, Grant D, Specht J, et al: RNA-Seq Atlas of Glycine max: A guide to the soybean transcriptome. BMC Plant Biology 2010, 10(1):160.

38. Fisher RA: A preliminary linkage test with Agouti and Undulated mice; The fifth linkage-group. Heredity London 1949, 3:229-241.

39. Kuo $\mathrm{CH}$, Ochman $\mathrm{H}$ : The extinction dynamics of bacterial pseudogenes. PLoS Genet 2010, 6(8).

40. Lerat $\mathrm{E}$, Ochman $\mathrm{H}$ : Recognizing the pseudogenes in bacterial genomes. Nucleic Acids Res 2005, 33(10):3125-3132.

41. Hirotsune S, Yoshida N, Chen A, Garrett L, Sugiyama F, Takahashi S, Yagami K-i, Wynshaw-Boris A, Yoshiki A: An expressed pseudogene regulates the messenger-RNA stability of its homologous coding gene. Nature 2003, 423(6935):91-96

42. Zheng DY, Frankish A, Baertsch R, Kapranov P, Reymond A, Choo SW, Lu YT, Denoeud F, Antonarakis SE, Snyder M, et al: Pseudogenes in the ENCODE regions: Consensus annotation, analysis of transcription, and evolution. Genome Res 2007, 17(6):839-851.

43. Svensson Ö, Arvestad L, Lagergren J: Genome-wide survey for biologically functional pseudogenes. PLoS Comput Biol 2006, 2(5):e46.

44. Wilhelm BT, Marguerat S, Watt S, Schubert F, Wood V, Goodhead I, Penkett CJ, Rogers J, Bahler J: Dynamic repertoire of a eukaryotic transcriptome surveyed at single-nucleotide resolution. Nature 2008, 453(7199):1239-1243.

45. $t$ Hoen PAC, Ariyurek $Y$, Thygesen $H H$, Vreugdenhil E, Vossen $R$, de Menezes RX, Boer JM, van Ommen GJB, den Dunnen JT: Deep sequencingbased expression analysis shows major advances in robustness, resolution and inter-lab portability over five microarray platforms. Nucleic Acids Res 2008, 36(21):e141.

46. Annous BA, Blaschek HP: Isolation and characterization of Clostridium acetobutylicum mutants with enhanced amylolytic activity. Appl Environ Microb 1991, 57(9):2544-2548

47. Qureshi N, Lolas A, Biaschek HP: Soy molasses as fermentation substrate for production of butanol using Clostridium beijerinckii BA101. J Ind Microbiol Biot 2001, 26(5):290-295.

48. Jesse TW, Ezeji TC, Qureshi N, Blaschek HP: Production of butanol from starch-based waste packing peanuts and agricultural waste. I Ind Microbiol Biot 2002, 29(3):117-123.

49. Ezeji TC, Qureshi N, Blaschek HP: Production of acetone, butanol and ethanol by Clostridium beijerinckii BA101 and in situ recovery by gas stripping. World J Microb Biot 2003, 19(6):595-603.

50. Li H, Ruan J, Durbin R: Mapping short DNA sequencing reads and calling variants using mapping quality scores. Genome Res 2008 , 18(11):1851-1858.

doi:10.1186/1471-2164-12-479

Cite this article as: Wang et al:: Single-nucleotide resolution analysis of the transcriptome structure of Clostridium beijerinckii NCIMB 8052 using RNA-Seq. BMC Genomics 2011 12:479.

\section{Submit your next manuscript to BioMed Central and take full advantage of:}

- Convenient online submission

- Thorough peer review

- No space constraints or color figure charges

- Immediate publication on acceptance

- Inclusion in PubMed, CAS, Scopus and Google Scholar

- Research which is freely available for redistribution

Submit your manuscript at www.biomedcentral.com/submit
Biomed Central 